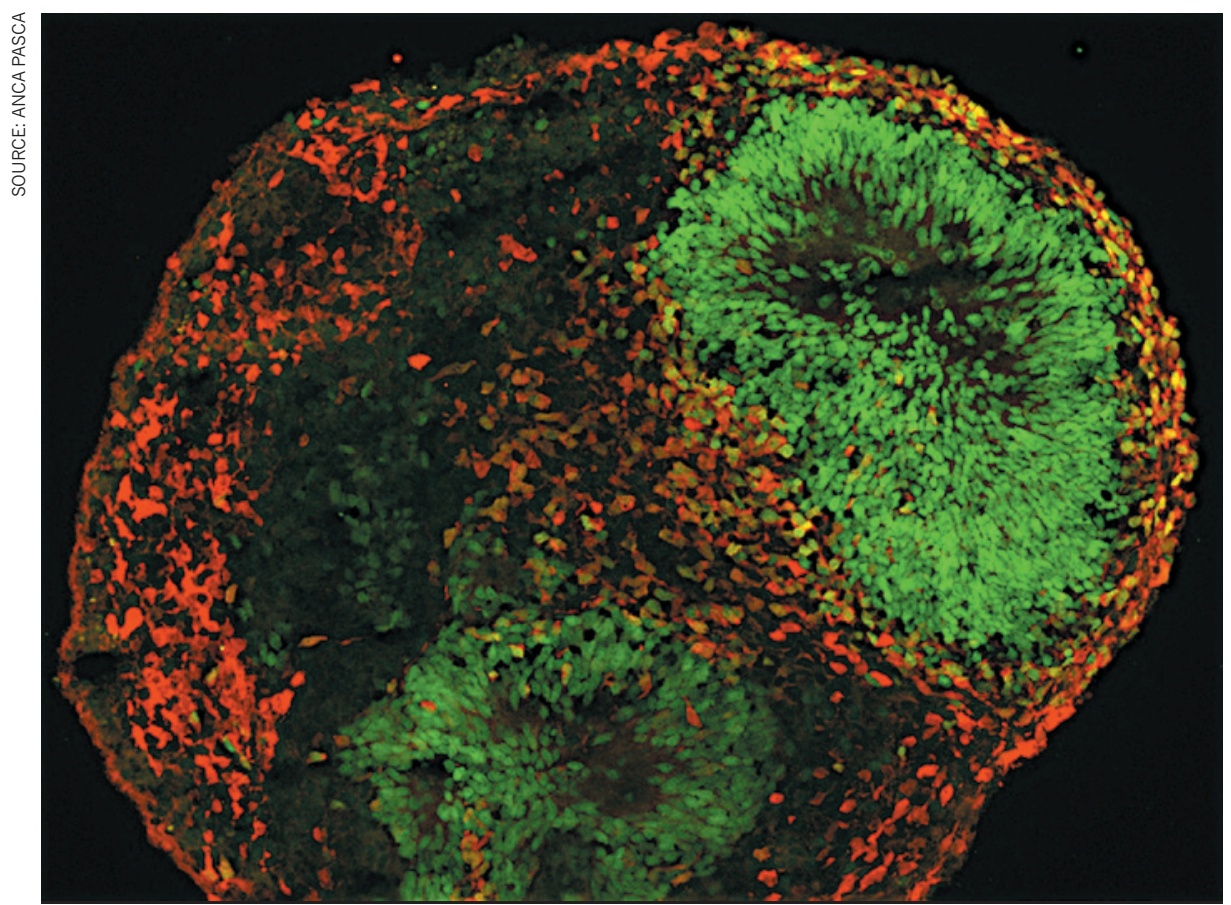

Pluripotent stem cells generated from patients' skin cells could be used to personalize brain therapies.

NEUROSCIENCE

\title{
Novartis reboots brain division
}

\section{After years in the doldrums, research into neurological disorders is about to undergo a major change of direction.}

\section{BY ALISON ABBOTT}

I $\mathrm{n}$ a sign that psychiatric-disease research is entering a new era, the pharmaceutical giant Novartis has hired an expert in neural circuitry, rather than pharmacology, to head its relaunched neuroscience division.

The appointment of 42-year-old Ricardo Dolmetsch, who has spent his entire career in academic research, signifies a radical policy shift for the company, as it moves away from conventional neurotransmitter research to concentrate on analysing the neural circuitry that causes brain diseases. The decision suggests Novartis is confident that after years of fruitless research in the field, revolutionary advancements in, for example, genetic and stem-cell technologies will pay dividends. The company intends to hire 100 new staff members for the department over the next 3 years. But the move is risky: even if it pans out, new drugs for common disorders such as schizophrenia could be decades away from reaching the market.

Dolmetsch, a former senior director at the Allen Institute for brain Science in Seattle,
Washington, who has also worked at Stanford University School of Medicine in California, says that his new role gives him access to previously unimaginable resources. "I had this idea that big pharma was a slow, plodding, conservative giant," he says. "I was surprised by the depth of science at Novartis."

An expert in autism spectrum disorder, he was also attracted by the prospect of contributing to the development of therapies - something that academic institutions are poorly equipped to do - particularly because one of his own sons has autism. There was "not much enthusiasm" for studying disease at the Allen Institute, which focuses instead on basic research into brain science, he says.

The World Health Organization estimates that one-quarter of people develop one or more disabling mental illnesses during their lifetime. Yet in the past few years, most of the world's major drug companies - Novartis included have abandoned psychiatric disease.

The company enjoyed some successes in the field in the 1960s and 1970s, including the development of clozapine, the first 
> of the 'atypical antipsychotic' drugs, and imipramine, the first of the tricyclic class of antidepressants. Both of these drugs spawned many look-alikes. But since these early breakthroughs, no radical improvements in therapy have emerged. For example, there is still no drug for the core symptoms of autism or for the particular cognitive deficits in schizophrenia that prevent most sufferers from holding down a job.

When Novartis closed down its conventional drug-discovery programme in Basel, Switzerland, last year (see Nature 480, 161$162 ; 2011)$, it was already planning to reopen with an entirely new approach at its global drug-discovery headquarters, the Novartis Institutes for BioMedical Research (NIBR) in Cambridge, Massachusetts. In its sights are neurodevelopmental diseases such as autism, schizophrenia and bipolar disorder, as well as neurodegenerative diseases such as Parkinson's and Alzheimer's disease.

Most current psychiatric drugs are based on the modulation of neurotransmitters, but the company's plan is instead to focus on connections between neurons, with the aim of addressing the precise neural circuitry that goes wrong in psychiatric disease, says Mark Fishman, the president of the NIBR. Genetics and environment both strongly influence how disease-causing circuits establish themselves.

Increasingly powerful genetic studies have begun to identify genes that might cause or contribute to the disorders (Cross-Disorder Group of the Psychiatric Genomics Consortium Nature Genet. 45, 984-994; 2013), and scientists can now efficiently model many of these defects in animals. With optogenetics - a technique introduced in 2005 that uses genetics in concert with light to activate or inhibit particular neurons - they can identify in living animals the neuronal circuits that the genes affect, and thus which genes could be targeted for therapy. In addition, stem-cell technologies now allow scientists to turn skin or hair-follicle cells from individual patients

"We'll begin
to see a lot of
clinical trials
within a decade
or so."
back into pluripotent stem cells, differentiate them into neurons and then study the connections that the neurons make with one another. Such induced pluripotent stem cells might one day aid the personalization of therapies to individual patients.

All of these technologies are young. Novartis is planning to collaborate closely with academic institutions to generate more genetic clues, build disease models and deepen the understanding of neural circuits in health and disease. The science will be published, says Dolmetsch, but Novartis will have first access to mutations and circuitry that are found to be linked to a disorder, for use in the company's drug-development programme. Novartis will also have the freedom to develop drugs around any target that emerges from genetic screens that it co-sponsors. "If academic groups feel they should patent targets, and if we contributed to the screen that revealed them, then we want the option to license those patents," says Dolmetsch.

Finding good targets, and moving from a target to a safe and effective drug, will not happen overnight, Dolmetsch stresses. "Everyone realizes that this is going to take a long time to bear fruit," he says. Steve Hyman, director of the Stanley Center for Psychiatric Research in Cambridge, Massachusetts, guesses that in five years - "if we all work hard enough" - it will be clear whether or not the new approaches are likely to be useful.

Hyman, who is an adviser to Novartis and a former director of the US National Institute of Mental Health, predicts that for diseases caused by a single gene, such as some rare but devastating forms of autism or intellectual disabilities, "we'll begin to see a lot of clinical trials within a decade or so".

The vastness of the task is keeping most big drug companies out of the game, although Roche, based in Basel, is committed to a similar approach, says the company's spokesman Stepan Kracala.

The European Commission is also trying to foster a collaborative approach through its Innovative Medicines Initiative. Two of its multimillion-euro programmes - EU-AIMS (European Autism Interventions - a Multicentre Study for Developing New Medications) and NEWMEDS (Novel Methods leading to New Medications in Depression and Schizophrenia) - combine different pharmaceutical companies and academic groups in projects that probe genetic and neural circuitry in autism, schizophrenia and depression.

Dolmetsch doesn't feel that his move to Novartis has distanced him from the intellectual centre of things. "The science at the NIBR is arguably better and certainly more rigorous than in most of academia," he says. ESEE EDITORIALP.141

Secrets of trial data revealed

\section{Records of side effects seen in clinical tests are missing from publicly available documents.}

\section{BY DANIEL CRESSEY}

$\mathrm{D}$ rug companies, regulatory bodies and research groups are in a deadlock over plans to throw open the vaults of sensitive data gleaned from clinical trials. But just as the battle seems to be coming to a head, a study (B. Wieseler et al. PLoS Medicine 10, e1001526; 2013) has revealed exactly why the restricted information could be so valuable to researchers.

According to the analysis, crucial trial information, such as mortality rates and serious side effects, is missing from much published data. But it can frequently be found in standard non-public documents prepared by industry, known as clinical study reports (CSRs). Missing information uncovered by the study includes details of depression symptoms in trials for antidepressant drugs, and details of heart attacks and strokes in diabetes-drug trials. "These are very, very important variables and outcomes," says the study's lead author, Beate Wieseler, head of the drug-assessment department at the Institute for Quality and Efficiency in Health Care in Cologne, Germany.

Wieseler strongly supports making all CSRs publicly available. "That is not an option, but a necessity," she says. "There should be no question that these documents be made available."

The issue of CSR access is shaping up to be a key sticking point in a major European push to make available more data from clinical trials. Drug companies send the reports to the European Union's European Medicines Agency (EMA) when they apply for licences to sell their products, and the agency has made clear that it would like to release the forms as part of a drive to increase transparency. It is currently drawing up a policy to that effect. Advocates of transparency say that such a policy will allow greater scrutiny of the benefits and harms of drugs, and will help researchers who are working on cures for diseases.

Some companies, including pharmaceuticals giants Roche, based in Basel, Switzerland, and GlaxoSmithKline in London, have already said that they will make their CSRs available to vetted researchers. But industry has pushed back against the wider moves to greater transparency. 